\title{
PROPIEDAD INDUSTRIAL PARA LA GESTIÓN DE CIENCIA, TECNOLOGÍA E INNOVACIÓN EN EMPRESAS ESTATALES CUBANAS
}

\author{
DULCE MARÍA CONTRERAS VILLAVICENCIO* \\ EVELIO SUÁREZ GUTIÉRREZ ${ }^{* *}$ \\ MARTA MILAGROS MORENO CRUZ ${ }^{* * *}$ \\ PASCUAL CORREA ÁLVAREZ ${ }^{* * * *}$
}

\section{INTRODUCCIÓN}

Cuando se examina el desarrollo de las empresas del mundo a cualquier nivel puede observarse cómo, por medio del conjunto de empresarios que las integran, se hacen irrepetibles y distintas, aun cuando pertenezcan a la misma rama o sector de la economía y empleen procedimientos, procesos, recursos materiales, humanos y financieros similares.

Se asevera en entornos científicos y académicos que la distinción empresarial radica hoy en los conocimientos que poseen los integrantes de las empresas, aptitudes innovadoras que comparten, la capacidad de adopción y diseño de estrategias en las relaciones de producción e intercambio que establecen en función de metas comunes.

* MSc. en Gerencia de la Ciencia y la Innovación, profesional del Derecho y profesora auxiliar a tiempo parcial de PI en la carrera Ingeniería Hidráulica de la Universidad Central "Marta Abreu" de Las Villas. Especialista en Mercadotecnia y gestora de Propiedad Intelectual de la Empresa de Investigaciones y Proyectos Hidráulicos de Villa Clara, Cuba. Contacto: dulcemaria@vc.hidro.cu

** MSc. en Gerencia de la Ciencia y la Innovación, economista y profesor auxiliar a tiempo parcial de la Universidad Central "Marta Abreu" de Las Villas. Especialista en Mantenimiento de Obras Ingenieras en la Empresa de Aprovechamiento Hidráulico de Villa Clara, Cuba. Contacto: evelio@vc.hidro.cu

*** Doctora en Ciencias Jurídicas, profesora titular Decana de la Facultad de Derecho de la Universidad de La Habana, Cuba. Contacto: marta.moreno@lex.uh.cu

**** Doctor en Ciencias Jurídicas, profesor titular, docente de la Facultad de Derecho de la Universidad Central "Marta Abreu” de Las Villas, Cuba. Contacto: pascualc@ uclv.edu.cu Fecha de recepción: 7 de mayo de 2017. Fecha de aceptación: 31 de mayo de 2017. Para citar el artículo: Contreras-Villavicencio, D. M.; Suárez-Gutiérrez, E.; Moreno-Cruz, M. M.; Correa-Álvarez, P. "Propiedad industrial para la gestión de ciencia, tecnología e innovación en empresas estatales cubanas", Revista La Propiedad Inmaterial n.o 23, Universidad Externado de Colombia, enero-junio 2017, pp. 147-169. DOI: https://doi.org/10.18601/16571959.n23.06 
La gestión de conocimiento, la inteligencia empresarial ${ }^{1}$, el benchmarking, la vigilancia y las prospectivas tecnológicas de mercado y propiedad industrial (en adelante, PI) constituyen algunas de las herramientas del sistema de innovación empresarial cubano $^{2}$, necesarias para lograr eficacia y eficiencia económica. Una de las fuentes estratégicas de gestión de la información tecnológica y comercial se encuentra en bases de datos públicas de patentes, boletines e informes anuales de instituciones nacionales, regionales e internacionales ${ }^{3}$.

La innovación, como factor decisivo para el logro del éxito económico, incide de manera directa en la gestión de carteras de tecnologías tangibles y el patrimonio intangible que se concibe en la contabilidad empresarial como activos cuando se apoya en derechos de propiedad intelectual, además de evidenciar competencias internas al asimilar el talento profesional de forma estratégica, difundir conocimientos útiles entre los miembros de la entidad y tener recursos con aptitud para generar considerables ingresos por su capacidad de aplicación y réplica en las negociaciones (Buitrago Díaz, 2009).

El Modelo Económico y Social Cubano reconoce la propiedad socialista de todo el pueblo sobre los medios fundamentales de producción como la forma principal en la economía nacional ${ }^{4}$, reto que asume el sistema empresarial estatal bajo el principio de la planificación socialista, responsabilidad social y medioambiental en interacciones conjuntas con factores locales y otros operadores del tráfico económico para que propicie la sostenibilidad de aportaciones de bienes y servicios de alto valor agregado derivados de la innovación en la solución de problemas de la realidad nacional y en la satisfacción de las demandas de la sociedad (PCC, 2011, 2016).

En la llamada sociedad o economía del conocimiento 5 y en el marco de la globalización económica imperante, resulta evidente la importancia de los bienes

1 Santos Riveras, "América, La propiedad industrial como mecanismo de contrainteligencia empresarial", trabajo presentado en el Taller Internacional de Inteligencia Empresarial y Gestión del Conocimiento en la Empresa (IntEmpres' 2000) que sesionó del 16 al 18 de noviembre de 2000, Hotel Meliá Cohíba, Instituto de Información Científica y Tecnológica (IDICT) del Ministerio de Ciencia, Tecnología y Medio Ambiente de la República de Cuba, La Habana, Cuba, 2000.

2 Orozco Silva, Eduardo, "La inteligencia empresarial como herramienta de la innovación", conferencia virtual, "Universidad para todos", ciclo de conferencias televisadas en el curso Nuevas Tecnologías, Clase n. ${ }^{\circ}$ 12, Instituto de Información Científica y Tecnológica (IDICT), La Habana, Cuba, 2007.

3 Romeu Lameiras, Eva, "La vigilancia tecnológica y la propiedad industrial en la gestión del nuevo modelo económico cubano", trabajo presentado en el VI Encuentro de Tecnología y Gestión del Conocimiento Tecnogesc 2014 que secionó del 22 al 23 de octubre, Convenciones Bolívar, Centro de Información y Gestión Tecnológica de Villa Clara, Santa Clara, Villa Clara, Cuba, 2014.

4 Actualización de los Lineamientos de la Política Económica y Social del Partido y la Revolución para el período 2016-2021, aprobados en el 7. ${ }^{\circ}$ Congreso del Partido en abril de 2016 y por la Asamblea Nacional del Poder Popular en julio de 2016, Lineamientos 1, 2. La actualización es continuidad de los Lineamientos aprobados en el $6 .^{\circ}$ Congreso. Ver Partido Comunista de Cuba, Lineamientos de la Política Económica y Social del Partido y la Revolución, n. ${ }^{\circ}$ especial, (Formato Digital ed.), Editora Política del ccrcc, La Habana, Cuba, 2011.

5 Se comparte el criterio de Núñez Jover, Jorge, La ciencia y la tecnología como procesos sociales. Lo que la educación científica no debería olvidar (2007), quien después de enunciar 
intangibles en el ordenamiento del comercio internacional con el uso y explotación de derechos de PI, situación que se refuerza con el Acuerdo sobre los Aspectos de los Derechos de Propiedad Intelectual relacionados con el Comercio (ADPIC; o TRIPS, por sus siglas en inglés), instrumento jurídico anexo al Convenio por el cual se crea la Organización Mundial del Comercio (oмc). Cuba se adhirió a dicho acuerdo en el año 1995, con la consecuente actualización en el sistema jurídico nacional (CE, 1999, 2002, 2012; Busaniche, 2015).

A pesar de lo descrito, son limitados e insuficientes en el país los conocimientos sobre PI y la adopción de estrategias por parte de las empresas en su gestión, que encuentran dificultades de aprendizaje y habilidades en el ejercicio a través de ciencia, tecnología y relaciones de intercambio (Ramírez Cabrera y Moreno Cruz, 1998; Horta Herrera et al., 2003; Lage Dávila, 2000, 2006, 2007, 2012, 2013, consultado 2015, Núñez Jover, 2004, 2007, 2010, 2014; Núñez Jover, Montalvo y Pérez, 2006; Suárez Arroyo, 2008; Contreras Villavicencio y Suárez Gutiérrez, 2014, 2016; PCC, 2016).

La situación descrita condicionó que se recordara el pensamiento transformador de Ernesto "Che" Guevara cuando advertía que la técnica se usa para domesticar a los pueblos pero también se puede poner al servicio de estos para liberarlos (Guevara de la Serna, 1970). En igual sentido se razonó por los autores como problema científico la interrogante siguiente: ¿̇cómo lograr la interrelación sistémica de la PI a través de la gestión de ciencia, tecnología y relaciones de intercambio que contribuya a la dinámica del patrimonio intangible de la empresa estatal socialista cubana?

Ante el cuestionamiento enunciado, se definió como objetivo general de este trabajo es el siguiente: proponer estrategias de PI a través de la gestión de ciencia, tecnología y relaciones de intercambio que guíen la dinámica del patrimonio intangible de la empresa estatal socialista cubana en el proceso de transformaciones del modelo de desarrollo económico y social actual.

El presente trabajo se estructura en tres partes que se concatenan. Refiere, en breve síntesis, criterios de diferentes autores que se utilizaron en la conformación del marco teórico referencial respecto a la PI en la empresa estatal socialista cubana para ubicarla por medio de la gestión de ciencia, tecnología e innovación; describe a continuación algunos elementos no exhaustivos de la investigación empírica que se aplicó en entidades nacionales, y en tercer lugar presenta alternativas viables para la adecuación de estos derechos en el entorno del sector objeto de estudio. Al final se formulan las conclusiones.

algunos de los argumentos que se emplean en diferentes fuentes y medios para reafirmar la idea de la "Sociedad del Conocimiento" y que en cierta medida o dentro de ciertos límites es correcta y permite aceptar la tesis, fundamentó cómo es verdad que el conocimiento es una fuente importante de poder, pero que en un mundo desigual, globalizado y dividido en cuanto a riquezas, este se convierte en una fuerza más en manos de los que poseen el poder económico y militar; que el $90 \%$ de científicos e ingenieros dedicados a $\mathrm{I}+\mathrm{D}$ del mundo son de los países desarrollados que dedican el $94 \%$ de los gastos a estos fines; y que los países no desarrollados, que albergarán el $75 \%$ de la población mundial en 2020, no pueden disfrutar a plenitud de la "Sociedad del Conocimiento". 


\section{i. Desarrollo}

\section{I.I. BREVE MARCO TEÓRICO REFERENCIAL SOBRE PROPIEDAD INDUSTRIAL EN LA GESTIÓN DE CIENCIA, TECNOLOGÍA E INNOVACIÓN EMPRESARIAL}

La generación, transformación, transmisión de información y conocimientos en la actualidad constituye la base sobre la que se construye la competitividad de personas y la productividad de las empresas a nivel social; implica un sistema de saberes para dar solución a problemas productivos, de servicio, administrativos y comerciales; lograr calidad, excelencia, valor agregado; introducir nuevas tecnologías; organizarse en redes de forma flexible y diversificada (Suárez Arroyo, 2008), así como establecer un sistema de planeación estratégica que permita anticiparse a los constantes cambios, lo que pareciera ser la clave del éxito empresarial (Pérez Almaguer, 2015).

El manejo y gestión de la información de patentes, diseños y otras modalidades de PI contenidas en bases de datos públicas, boletines e informes de oficinas administrativas, constituyen fuentes para el aprendizaje continuo como verdaderos programas de capacitación tecnológica a trabajadores de las distintas ramas de la economía; superación profesional que garantizaría el aumento de capacidades y habilidades en relación con tecnologías de las ramas del saber en las que participan, incentivación de los procesos de innovación y toma de decisiones estratégicas (Matheus Osechas, 2014).

De acuerdo con Xing, Ge y Song (2016), ciencia, tecnología y pensamiento innovador tienen potencial para lograr productividad empresarial, mejorar procesos productivos, creación de bienes y servicios para la sociedad donde encuentran valor los derechos de PI, que, aunque de naturaleza inmaterial, tienen valor de uso y valor, base del desarrollo organizacional en la era de la economía del conocimiento. Según Títu et al. (2016), deberá tenerse en cuenta la rapidez con la que salen al mercado los nuevos productos, marcas, modelos y otros resultados a partir de la innovación y el proceso diario de creación. En Cuba, al igual que en otros países, se ajustan políticas para guiar la comercialización estratégica con activos intangibles (De León y Fernández Donoso, 2016).

En igual sentido, Vicente Oliva, Martínez Sánchez y Berges Muro (2015) aseguraron que para desarrollar con éxito proyectos de investigación, desarrollo e innovación se requiere que la empresa pueda transformar e implementar conocimiento adquirido del exterior, y argumentaron que las mejores prácticas se relacionan con la capacidad de absorción de conocimientos o habilidades para convertirlos en otros nuevos que la organización pueda utilizar con fines comerciales.

Los aspectos señalados en el párrafo precedente se consideran oportunidades para alcanzar competitividad, por lo que su instrumentación como política de ciencia y tecnología nacional debería regularse desde la base, en las empresas; ello permitiría el ejercicio sistemático de esos saberes como prácticas honestas de 
asimilación de información externa que facilitaría la creación de conocimientos de generación interna con ventajas en la productividad de los procesos de producción, nuevos o mejorados bienes y servicios (Rengifo García, 2011; Contreras Villavicencio, 2015; CEPAL, 2016).

La PI como mecanismo de competitividad tiene enfoque integral y estratégico, sus impactos en la planeación empresarial se mueven entre la protección y difusión de conocimientos como funciones básicas de su sistema (UE, 2008; Pitkethly, $2010)^{6}$.

La institución de referencia se manifiesta mediante registros públicos de tecnologías, diseños, marcas y otros signos distintivos, secretos empresariales, represión contra la competencia desleal, así como a través de la comercialización de diferentes modalidades de PI que se establecen mediante figuras contractuales como licencias, franquicias, alianzas estratégicas y otros acuerdos con centros de investigación, producción u otros operadores del comercio (Ompi, 2011 y 2013; Mora, 2015).

El lineamiento 110 de la Política Económica y Social del Partido y la Revolución proyecta la voluntad de fortalecer, junto a las capacidades de prospección y vigilancia tecnológica, la protección de la PI en Cuba y los principales mercados externos (PCC, 2016).

El perfeccionamiento del lineamiento de referencia promueve la creatividad intelectual en el proceso consciente de desarrollo de actividades intensivas y complejas que integran la gestión de información externa, asimilación y creación de nuevos conocimientos con valor agregado que estimulan la innovación y que resultan estratégicos para la empresa estatal socialista en el interés de elevar la competitividad y perfeccionar sistemas de relaciones de intercambio que faciliten la comunicación en alianzas estratégicas de colaboración científico-técnica y comerciales.

En esos procesos de gestión y toma de decisiones respecto al uso y explotación de bienes intangibles patrimoniales empresariales se requiere promover de manera conjunta las dos funciones básicas de protección y difusión, como regla obligatoria para que en todas las actuaciones se prevea y garantice responsabilidad con los resultados del conocimiento, los impactos o beneficios a nivel de la sociedad y se ofrezcan soluciones medioambientales a la realidad práctica local y nacional.

En la actualidad, la empresa estatal socialista o de propiedad de todo el pueblo se convocó a proyectarse hacia una mayor flexibilidad en su desempeño para lograr eficiencia, productividad, competitividad y mayores aportes a la sociedad, lo que implicaría alcanzar estándares de calidad en la organización de sus procesos, procedimientos y la implementación de actuaciones conscientes que articulen potencialidades internas con el uso racional de los bienes que se administran y el despliegue de capacidades intelectuales en la obtención de resultados del conocimiento. 
A través de la empresa estatal socialista se expresa el carácter de la propiedad social sobre los medios fundamentales de producción y la apropiación del producto social con arreglo al trabajo en Cuba, donde, dada la estructura demográfica y cultural de la población y la escasez de recursos naturales, la producción tiene que ser de alto contenido tecnológico, generando productos de alto valor agregado e innovación.

La competitividad, en cuanto reto de la empresa estatal socialista, es expresión y a su vez continuidad de la organización en función de lograr sostenibilidad, eficiencia, rentabilidad económica y del capital humano, es satisfacer las necesidades del mercado y presupone cumplir requerimientos internos de autosuperación, emprendimiento en su entorno y en todos los ámbitos en función de la sociedad (Pérez Almaguer, 2015).

Desde la perspectiva anterior, puede considerarse a la competitividad como sinónimo de capacidad, aptitud, idoneidad de sujetos naturales y jurídicos para gestionar de manera estratégica el trabajo intelectual en la resolución de problemas de la realidad y el establecimiento de redes de intercambio sostenidas. Este comportamiento consciente debe ser la regla obligada que incentive valores de colaboración, integración, creatividad colectiva y participativa enfocada en los clientes para satisfacer las demandas de la sociedad.

El Decreto 281 de 2007, Reglamento para el Sistema de Dirección y Gestión Empresarial, ha sido objeto de varias reformas hasta la actualidad (CM, 2014). Reafirma el régimen del perfeccionamiento empresarial como norma de comportamiento de la empresa estatal socialista cubana, distingue la persona jurídica de la natural, e instituye atribuciones y deberes organizacionales que separan las que corresponden al director general, lo que significa responsabilidad colectiva e individual de empresarios como integrantes de la entidad y las de su representante legal para alcanzar metas o fines con los medios que disponen respecto al encargo social.

Cabe señalar que el tratamiento del referido reglamento respecto a la PI es potestativo, solo indica que se garantice su gestión. En las primeras versiones tomó en cuenta la PI relegando al derecho de autor (Morejón Borjas, 2012), que aunque no es objeto de análisis en el presente trabajo, debe tenerse en cuenta para la protección de los programas de ordenadores y otras de sus modalidades, como establece el ADPIC, que pueden utilizarse de forma creativa y agregar valor en el comercio.

Esta disposición que se referencia remite a otras legislaciones específicas a pesar de establecer la integralidad sistémica del proceder empresarial estatal. Su actualización del año 2014 respaldó conceptos como activos intangibles, patentes, derecho de autor, licencias, marcas y otros, de aplicación a la industria, el comercio y los servicios en $\mathrm{Cuba}^{7}$ (Vázquez de Alvaré y Moreno Cruz, 2013).

\footnotetext{
7 Ver los decretos-leyes 203 de 1999, 228 de 2002 y 290 a 292 de 2012, que expresan la voluntad del Estado cubano de armonización de las normas internacionales al sistema jurídico y modelo económico y social cubano en materia de PI en sus modalidades de marcas y otros signos distintivos y tecnológicas para todas las ramas.
} 
El referido Decreto 281 de 2007 enfatiza en la instrumentación del sistema de innovación a través de la divulgación del banco de problemas y soluciones que se logran por la acción destacada de los miembros de la Asociación Nacional de Innovadores y Racionalizadores (ANIR) y el Fórum de Ciencia y Técnica para la introducción y generalización de resultados en la producción y los servicios de cada entidad, donde se involucra a todos los creadores de manera voluntaria, de acuerdo con una práctica propia del sistema social cubano.

A la ANIR se afilian personas vinculadas o no a centros laborales; disfruta de la anuencia del Estado, reconoce la actividad creadora de trabajadores y jóvenes; constituye un mecanismo de respuestas o soluciones a problemas de la producción o los servicios ante la escasez e imposibilidad de acceder a piezas de repuestos y otras tecnologías; esto último debido al férreo bloqueo económico, financiero y comercial impuesto por Estados Unidos a Cuba por más de 50 años, y a la necesidad de desarrollo de la sociedad en beneficio del pueblo.

Si bien el movimiento de innovadores y racionalizadores tiene una regulación especial en el país, estas creaciones no tienen que cumplir requisitos de invenciones y otras modalidades de PI vinculadas con soluciones técnicas; la novedad y utilidad que se requiere solo tiene alcance a nivel de empresa, donde se registran, examinan y dictaminan los resultados que aportan beneficios económicos, sociales, para la defensa y el orden interno mediante resolución del director (CM, 1984; Peruchena, 2015).

Tanto la ANIR como el Fórum de Ciencia y Técnica son instituciones autóctonas que surgieron en el proceso revolucionario cubano, y constituyen la identidad histórica de la labor abnegada de trabajadores y jóvenes que acogieron la tarea de innovar y dar soluciones técnicas a problemas tecnológicos por la necesidad de proyectar y materializar el desarrollo del país sobre la base de la preparación o instrucción del pueblo.

Concordante con los aspectos expuestos, Fidel Castro Ruz, líder histórico de la Revolución cubana, en la clausura del xI Fórum reconoció la importancia y necesidad de la protección de los resultados de la ciencia, y expresó que esto podía representar beneficios económicos para el país y además evitar litigios con otros países hermanos; al respecto afirmó: "Ya estamos viendo la ciencia convertida no solo en algo que ayuda a salvar vidas, a aliviar el dolor, a resolver problemas muy humanos, sino que puede significar importantes ingresos para el país. Claro, todo eso hay que patentarlo, protegerlo dentro de lo posible; apoyarnos en las organizaciones mundiales" (Castro Ruz, 1996).

Sin embargo, a pesar de existir mecanismos relativos al Sistema Interno de Propiedad Industrial (SIPI) (CITMA, 2003), que incluye pautas rectoras, lineamientos metodológicos comunes que rigen conductas y acciones de actores sociales en su ámbito interno y relaciones frente a terceros, así como una guía para diagnósticos y controles, en la práctica este no alcanza su efectividad en la integralidad sistémica del perfeccionamiento empresarial como herramienta de la innovación y buenas 
prácticas de su uso en el comercio. En efecto, los estudios institucionales corroboran insuficiencias de gestión y estrategia, elementos que sirvieron de base a la nueva política de este sistema, vigente desde el año 2015.

En el entorno empresarial, la PI constituye una herramienta de innovación consciente y un mecanismo para evitar la ocurrencia de conductas inapropiadas. Triana Cordovi $(2003,2012)$ señala como situaciones negativas en el interior de las empresas cubanas: el hecho de que muchas veces el pago por el conocimiento solo se traduce en reconocimiento y prestigio; el que en ocasiones líderes de estas entidades presentan ideas innovadoras como suyas y privan a autores miembros de la organización de la única recompensa a la que pueden aspirar; el reconocimiento social o del colectivo laboral generándose actitudes de retraimiento por parte de autores ante semejantes actos de piratería.

En igual sentido, dicho autor reflexiona sobre los directivos o gerentes que crean de manera deliberada asimetrías en el flujo de información con el propósito de incrementar de forma artificial su poder, y expresa que esa es una actitud dañina porque transmite valores y una cultura que se aleja de las líneas maestras del diseño histórico y actual de las políticas sociales cubanas.

Sobre el retraimiento y la inhibición, asegura que en ocasiones se asiste a comportamientos que convierten el conocimiento empresarial en monopolio de sus poseedores que evitan compartirlo como forma de hacerse imprescindibles para la entidad; y reflexiona sobre la necesidad de potenciar las fortalezas de la empresa, enfocándose en el logro de una cultura dirigida a compartir o transmitir de manera estratégica el conocimiento que acumulan sus trabajadores.

La presencia de diversos operadores del comercio en las relaciones económicas y la apertura a la inversión extranjera en Cuba hacen de los derechos de PI un instrumento necesario en el entorno nacional, no solo para las principales industrias sino para todo el sistema empresarial, y de especial relevancia en el sistema de dirección y gestión organizacional como actividad administrativa con alcance fuera de ella hasta el entorno global. Esto implica la observancia obligada de tratados, arreglos y acuerdos que rigen en el comercio internacional.

Los razonamientos precedentes constituyen aspectos que es preciso tener en cuenta en las reglas de comportamiento empresarial, por lo que pudiera valorarse dentro del régimen del perfeccionamiento e instituirse como una atribución de la empresa y su director general la planificación estratégica de la PI a través de la gestión de ciencia, tecnología, relaciones de intercambio y responsabilidad patrimonial de los derechos sobre bienes intangibles en función del desarrollo económico, social y medioambiental que promueva inclusión y colaboración en la sociedad cubana (Kalanje ${ }^{8}$, consultado en 2016).

La formación de redes multidisciplinarias de intercambio como vía para convertir a competidores o empresas similares en aliados constituye una forma de 
propagar la creatividad, impulsar el desarrollo y al mismo tiempo evidenciar ante la sociedad cuáles son las empresas innovadoras, estimulando a las que aún no lo son (Contreras Villavicencio, Suárez Gutiérrez y Recarey Morfa, 2016).

En ese mismo sentido, las alianzas entre universidades y empresas, así como la participación de factores de la localidad, o sea, los gobiernos territoriales, constituyen reservas importantes cuya generalización significaría un verdadero avance en el proceso de innovación (Gouvea, Kassicieh y Montoya, 2012; Kim, Kim y Yang, 2012; Strand y Leydesdorff, 2012).

Puesto que la economía del conocimiento fundamenta la conexión directa del mundo científico con el empresarial (Lage Dávila, consultado en 2015), se requiere que estas entidades sean de alta tecnología, estando llamadas a arbitrar entre la innovación y su valor económico, donde es vital la visión de los líderes. Las decisiones de Fidel Castro al crear el Polo Científico de la Biotecnología y la Universidad de las Ciencias Informáticas (UCI) son ejemplos que lo evidencian.

En el país, según el autor de referencia, se necesita un grupo de entidades del aparato empresarial, así sea pequeño, que estimule un mayor nivel de inversión en investigación y desarrollo, incluyendo la financiación empresarial de proyectos conjuntos con instituciones académicas o universitarias, y que promueva que se investigue y produzca en función de la demanda no solo doméstica, y que se exploren esquemas de negociación interna y externa innovadoras.

El autor en cita reflexionó sobre la carta que dirigiera el "Che" a Fidel Castro en 1965 refiriéndose a la falta de conexión entre ciencia y economía en la Unión Soviética debido al estancamiento de la técnica en la inmensa mayoría de los sectores económicos, y cómo en la Academia de Ciencias de ese país existían engavetados centenares de proyectos de automática que no podían ser llevados a la práctica porque los directores de las fábricas tenían que dedicarse a cumplir sus planes de producción.

El no aprovechamiento de las potencialidades de los soviéticos hizo que se establecieran alianzas con otros países para obtener transferencia de tecnología en materia de radares, computadoras, máquinas-herramientas y semiconductores. La producción y el transporte de petróleo y gas de los países de la Unión eran prioridades que fueron desactivadas por software con programaciones nocivas de manera intencional (Castro Ruz, 2007).

\section{I.2. FundaMENTOS EMPÍRICOS DE INDAGACIÓN}

El desarrollo de la investigación se orientó sobre la base de un enfoque dialéctico, mixto o multimodal con apoyo en la observación científica participativa y experiencia vivencial, entre otras herramientas comunes a esos procesos; métodos del nivel teórico, inductivo-deductivo, análisis-síntesis, abstracción-concreción, histórico-lógico entre otros y empíricos; encuestas, entrevistas, listas de chequeo, etc. (Mantilla Cárdenas, 2001, 2003; Hernández Sampieri, Fernández Collado y 
Baptista Lucio, 2006; Fabelo Corzo, 2009; Villabella Armengol, 2012; Moreno Linde, 2015).

En correspondencia con lo anterior, se declara que constituye una investigación socio-jurídica, enfocada hacia la propuesta de solución del empleo del derecho de PI en un entorno que se llamó a ser dinamizador de la sociedad cubana más allá de tendencias normativistas (Fernández Bulté, 2008; Pérez Hernández, 2012).

Se tomó como población a las empresas estatales cubanas de bienes y servicios, y el criterio que se siguió para la selección de la muestra investigativa tuvo en cuenta organizaciones con Sistemas Internos de Propiedad Industrial (sIPI), entidades asociadas a la Cámara de Comercio de la República de Cuba que realizan actividades de importación o exportación, y empresas líderes del país con práctica sistemática en el uso y explotación de modalidades de dicha institución en el comercio internacional.

Se razonó la selección a partir de pesquisas preliminares que permitieron conocer que no todas las empresas en proceso de perfeccionamiento tenían SIPI; aquellas que lo implantaron procedieron al registro de marcas y otros signos distintivos sin otras gestiones adicionales, en donde algunos registros que se relacionan con el nuevo conocimiento se tramitaron por la vía del derecho de autor; en otras se afiliaron solo al registro por la vía de la ANIR, no sobre patentes o diseños industriales u otras modalidades de PI.

Los primeros resultados del estudio se orientaron al universo de la propiedad intelectual en las estrategias de dirección y gestión empresarial. Entre los años 2003-2005 se aplicó a un caso único del territorio villaclareño, enfocándose en la dirección estratégica para gestionar la PI de la entidad investigada que recién se iniciaba en el perfeccionamiento empresarial y se proyectaba en la integración de este sistema; se elaboró un programa de capacitación segmentado que se ajustara a las actividades que ofrecían valor para su desarrollo, y se aplicó en diseños de ingeniería, trámites de búsqueda de interferencias, registro de marcas y otros signos; se obtuvieron registros por la vía del derecho de autor para obras informáticas en el marco del empleo laboral, pero no se comercializaron bajo contratos de licencia.

Entre 2006-2008 se incluyeron empresas homólogas del mismo grupo a nivel nacional, se verificaron los resultados que arrojaron situaciones similares respecto de las tramitaciones de marcas y otros signos distintivos como identidad organizacional sin otras acciones estratégicas que demostraran actuaciones conscientes en el comercio, insuficientes gestiones ante la oficina administrativa relativa a la solicitud de servicios de búsqueda de información, vigilancia tecnológica y registros legales; algunos resultados profesionales se tuvieron en cuenta como obras literarias y productos informáticos por la vía del derecho de autor, otros trabajaron la ANIR como vía de reconocimiento y estimulación, la mayoría de las innovaciones que se presentaron al Fórum de Ciencia y Técnica no se protegieron. 
Los resultados que anteceden justificaron la ampliación de la muestra que se investigó, a partir del año 2009 se extendió a un grupo de empresas estatales importadoras y exportadoras de diferentes sectores económicos asociados a la Delegación Centro de la Cámara de Comercio. Se corroboró entonces que no todas tenían sipi, encargándose la entidad nacional de las tramitaciones y registros correspondientes en materia de marcas, algunas tenían una organización de la ANIR activa, otras menos dinámicas, no se identificaron registros por la vía de modalidades tecnológicas de PI, se evidenciaron registros de derecho de autor y se mostró exigua la referencia de estos derechos en el registro contable financiero.

En eventos internacionales de información (INFO) se pudo constatar con especialistas y directores de Centros de Información y Gestión Tecnológica (CIGET) adscritos al Ciтma la existencia de dificultades que afrontan sus consultoras para internalizar la PI en las empresas y se apreció el escaso dominio en el manejo de bases de datos de patentes y servicios que ofrece la Oficina Cubana de PI (OCPI). Lo anterior demostró la necesidad de reforzar la labor divulgativa y de acompañamiento de las instituciones estatales en colaboración con la OMPI a través del programa Centros de Apoyo a las Tecnologías de la Información (CATi) que se extiende por el país en función de la innovación.

En fecha posterior se incluyeron entidades líderes del país que usan y explotan derechos de PI para conocer mejores prácticas, aciertos y desaciertos en su gestión. Se aplicaron listas de chequeo, entrevistas en profundidad y de oportunidad que permitieron obtener información en el menor tiempo posible en función de identificar aquella que pudiera resultar relevante para su adecuación en la empresa estatal socialista cubana. Los resultados evidenciaron la existencia de diversos manejos de modalidades y estrategias de dichos derechos sobre bienes intangibles patrimoniales y que de manera indistinta establecen alianzas con centros académicos, de investigación y distribuidores, siendo común el uso de acuerdos de confidencialidad en procesos de creación y comercialización.

Las empresas utilizan diversas figuras contractuales en la comercialización de los derechos de PI y para ello se auxilian con el servicio de revisión de contratos en la OCPI, procedimiento obligatorio solo en casos de contratos vinculados con la inversión extranjera, nacional y en casos de contratos de colaboración científicotécnica; no obstante, en las empresas se consideró válido en otras esferas, por el desconocimiento que existe sobre esta materia, gestionando asimismo herramientas de innovación como la vigilancia e inteligencia empresarial con utilización de bases de datos de patentes y boletines de oficinas administrativas.

En igual sentido, pudo conocerse la coincidencia de criterios en empresarios en cuanto al reconocimiento de las actuales limitaciones del empleo de la PI, su importancia para la competitividad de la empresa estatal socialista cubana, la necesidad de capacitar, crear equipos multidisciplinarios para la toma de decisiones y de financiación para la protección y defensa de derechos de PI en Cuba y en el extranjero. 
A partir de los resultados obtenidos, los autores de este trabajo se afiliaron a los criterios que expresara Lage Dávila (consultado en 2015) en cuanto a que cualquier empresa estatal cubana pudiera tener un determinado componente de I+D, y a basar su estrategia en la introducción de productos novedosos, que no existen hoy o que sustituyan otros productos líderes. También se consideraron referentes las posiciones de Mora (2014) y De Las Heras et al. (2015) en cuanto a la gestión contable financiera.

\section{i.3. Alternativas en las estrategias de propiedad industrial \\ A TRAVÉS DE CIENCIA, TECNOLOGÍA E INNOVACIÓN EN EMPRESAS ESTATALES CUBANAS}

El diseño de estrategias de PI que se propone para la empresa estatal cubana, como alternativa viable para la gestión de dicha institución, se analiza respecto del conjunto de actuaciones de la organización como un todo que proyecta alcanzar metas y su visión de futuro en función de la obtención de ingresos adicionales, ello mediante la incorporación en sus relaciones jurídicas económicas, bienes con valor agregado que se derivan de la información, conocimiento e innovaciones de sus trabajadores.

En ese sentido, a continuación se describen algunas alternativas que se ajustan a la dinámica del perfeccionamiento empresarial e integralidad sistémica de la dirección y gestión con estrategias de conducción de la ciencia, tecnología e innovación, todo esto en función del desarrollo económico, la satisfacción de demandas del pueblo y la responsabilidad con el medio ambiente; aspectos esenciales del modelo económico y social cubano para alcanzar prosperidad y sostenibilidad (Contreras Villavicencio y Suárez Gutiérrez, 2016).

- Debería reconocerse a los promotores de ideas productivas o innovadoras empresariales con independencia del curso de estas en el proceso de toma de decisiones estratégicas de la entidad, esto para incentivar en los colectivos internos la predisposición al cambio, comportamientos éticos y buenas prácticas de inteligencia empresarial como regla que discipline el respeto por todos aquellos trabajadores que aportan al cumplimiento de las metas empresariales y de los compromisos que asumen con la sociedad.

- Si bien a nivel internacional la idea no es registrable, la presente propuesta aprovecha la experiencia del registro de la ANIR para que se perfeccione en cuanto a la identidad de la creación o idea productiva con su/s promotor/es en función de que se reconozcan las raíces de las soluciones, que se participe junto a los desarrolladores en la gestión de los proyectos y se tenga en cuenta dicha participación en el trabajo intelectual y la legitimidad de la creación. Se garantiza así que las personas se comprometan, y que se estimule la 
masividad y colaboración como principios que logren materializar los fines supremos necesarios en el modelo de desarrollo económico y social cubano.

En igual sentido, sería necesario valorar las iniciativas y criterios de los promotores de ideas que se incluyan en el proceso de trabajo intelectual como autores cuando se legalicen los resultados obtenidos, se reconozcan e incentiven con los estímulos que se establezcan para lograr que se comprometan a continuar con aportaciones a la sociedad.

Lo anterior implicaría desaprender viejas prácticas de captación de creaciones intelectuales que por lo general aparecen de manera espontánea por iniciativa de trabajadores, para proceder a aprender nuevos comportamientos, procesos ordenados en la cadena de valor a ciclo completo. Lo anterior significa investigación, desarrollo e innovación, hasta la comercialización, bajo los principios fundamentales de la planificación y distribución socialista que posibilite su integración en la cultura organizacional de manera sostenible.

- En el entorno de las relaciones externas a la empresa estatal creadora frente a terceros le es preciso aplicar la PI, lo que significa que como regla se establezca la conservación de la propiedad del bien intangible como patrimonio empresarial para licenciarlo y procurar así ingresos sucesivos y seguimiento a las mejoras que se logren atendiendo a la razón social aprobada para dichas entidades, y que se puedan hacer transferencias a otros factores locales $\mathrm{u}$ operadores del comercio que promuevan la colaboración mutua o el emprendimiento mediante alianzas donde se empleen instrumentos jurídicos conforme a las flexibilidades que ofrece la legislación contractual vigente en Cuba.

El establecimiento de estas relaciones jurídicas entre partes aliadas pudiera aprovechar las modalidades de convenio en procesos de colaboración con instituciones u otras entidades en la gestión de proyectos a ciclo completo de innovación y los contratos económicos en transacciones frente a terceros operadores del comercio.

- Se hace necesario conformar equipos multidisciplinarios para el proceso de trabajo intelectual empresarial. En el orden reglamentario pudiera constituirse en una nueva forma de organización del trabajo que permita aplicar la integralidad de los sistemas internos de propiedad intelectual que incluya sus dos ramas, PI y derecho de autor y el el establecimiento de buenas prácticas referidas a las herramientas de innovación como vías honestas para el manejo de fuentes de información, vigilancia, prospectivas tecnológicas, inteligencia empresarial y benchmarking utilizando las técnicas del marketing en la gestión del negocio, control de resultados, protección-difusión y empleo de instrumentos jurídicos. 
- Se requiere la valuación o valorización de activos intangibles que pertenecen al patrimonio empresarial a través de los métodos más convenientes, así como en procesos de investigación, desarrollo e innovación, todo lo cual resulta necesario a la hora de concertar contratos de transferencia tecnológica que incluyan derechos de PI y también al aportar dichos bienes intangibles como capital en negociaciones con otras empresas. Esto es relevante para el país a partir del fomento de la inversión extranjera.

- Resulta pertinente la consulta permanente de la información de patentes por parte de las empresas desde el propio proceso de investigación científica, a la hora de la protección de los resultados de innovación a través de las diferentes modalidades de la PI y en los procesos de transferencia tecnológica.

- Las empresas requieren implementar la vigilancia permanente de sus activos y evitar que terceros ostenten derechos intelectuales que no les corresponden, aspecto que puede implementarse a través del uso eficiente de las oposiciones en los procedimientos de concesión de patentes, marcas y otras modalidades de PI, la observancia del uso de los derechos de dicha institución y la presentación de procesos de infracción de derechos en los casos procedentes.

- Es necesaria la realización de contratos de investigación científica entre universidades, centros de investigación, empresas y otras entidades, en los cuales se identifiquen con claridad las cláusulas de PI necesarias para definir la titularidad de los derechos de PI derivados de las investigaciones al igual que otros elementos necesarios en esta materia. Asimismo, en los casos en que se convenga una cotitularidad resulta imprescindible realizar acuerdos de cotitularidad en los cuales se definan los pactos necesarios para el registro, administración y ejercicio conjunto de los derechos.

- En igual sentido, se considera relevante adecuar la planificación respecto a elementos de mercadotecnia para la gestión de nuevos atributos en la creación o mejora de productos/servicios en relación con la identidad e imagen, el posicionamiento y las decisiones del comprador o consumidor, por cuanto implican vías y formas de comunicación de mensajes, estudios de inteligencia comercial respecto a proveedores o distribuidores, y desarrollo de marcas comerciales, entre otras posibilidades que permitiría el intercambio con una cartera de negocios distintiva en razón de los derechos sobre bienes intangibles patrimoniales que la integran.

- Es esencial, en materia de mercadotecnia, el estudio de la demanda para diseñar estrategias en esa materia y de PI con enfoque objetivo a la satisfacción de necesidades básicas, y regular la presencia del mercado en función del poder adquisitivo de los segmentos de población a los que se dirigen los esfuerzos en las condiciones concretas de Cuba y en los nichos de mercados extranjeros a los que se dirijan las exportaciones para alcanzar precios justos respecto a la demanda, no a la competencia. 
- Resulta ventajoso el establecimiento de la organización del trabajo por proyectos con utilización de la PI, buenas prácticas de uso de sus modalidades en la gestión de la ciencia, tecnología e innovación empresarial, en la formación de redes de relaciones en alianzas estratégicas con universidades para el desarrollo local, nacional y la exportación, a través de la identificación y reconocimiento de colectivos innovadores por sus resultados concretos, el valor agregado del trabajo intelectual derivado de los ingresos, y los impactos o beneficios a la sociedad y el medioambiente de manera sostenible.

- Es importante el empleo de instrumentos jurídicos como los contratos de licencias para el uso de modalidades de las ramas de propiedad intelectual que, por un lado, garanticen la propiedad empresarial de sus derechos patrimoniales sobre bienes afectos por los fines con la patrimonialidad de estas entidades estatales, y por el otro, aseguren la cesión parcial o transferencia que permita visualizar la generalización y cuota de mercado que se alcanza con los bienes legitimados por estas organizaciones.

En estos contratos se deberán definir cláusulas importantes referidas al tipo de licencia, el territorio, los derechos cedidos o licenciados y su alcance, el precio de la tecnología cedida y su forma de pago, bien por medio de una suma alzada, royalties, o de la combinación de ambas formas, las vías de protección de los perfeccionamientos y mejoras a las tecnologías, cómo comprobar o auditar los ingresos obtenidos por el licenciatario, la vigencia del contrato, la no inclusión de cláusulas restrictivas, ente otros elementos.

En correspondencia con lo anterior, también se reflejaría la difusión o generalización al visualizar los segmentos de mercado que se atiendan por territorios locales a nivel nacional y en el extranjero, regalías que se obtengan en cada período y contribuciones fiscales que correspondan, informaciones de utilidad a todos los efectos que implica el control interno y la supervisión de los intereses en juego respecto a las responsabilidades que asume la empresa estatal socialista cubana con dichos bienes del patrimonio intangible empresarial frente al Estado y el pueblo (Suárez Gutiérrez y Contreras Villavicencio, 2016).

La cesión total de los derechos por parte de entidades estatales a terceros podría limitar su competitividad y sostenibilidad económica en función de los compromisos asumidos ante la sociedad de ampliar las aportaciones con sostenibilidad. Adoptar estas estrategias de manera deliberada pudiera considerarse desde la economía política como una manifestación pasiva de robo de cerebro, propiciada por la organización encargada de protegerlos, gestionarlos y acrecentarlos en función de su verdadero dueño, el pueblo, y que incluye a sus propios trabajadores, considerándose que deberían instituirse estos actos de transmisión como violatorios del régimen económico y social en el ordenamiento penal cuando afecten los intereses a largo plazo el desarrollo y bienestar de la sociedad o al medio ambiente. 
- La perspectiva de negociaciones y transferencia de derechos sobre bienes del patrimonio intangible empresarial respecto a la exportación e importación debe establecerse sobre la base de un equilibrio a nivel empresarial, bien a través de proyectos de inversión interna, de colaboración local o extranjera para la mejora continua de nuevas tecnologías que se incorporen a los procesos de producción material e intelectual, y por otra parte el incremento del valor agregado de la creatividad e innovación para salvaguardar la soberanía tecnológica y cultural de la nación.

\section{ConCLusiones}

Las consideraciones sobre las estrategias de PI a través de la gestión de ciencia, tecnología, innovación y relaciones de intercambio en función de dinamizar el patrimonio intangible de la empresa estatal socialista cubana, en el régimen del perfeccionamiento de estas entidades, pudieran contribuir a la planificación, disciplina y armonización de los intereses estatales con operadores nacionales y extranjeros del comercio acorde al sistema jurídico cubano en el proceso de transformaciones del modelo de desarrollo económico y social que se construye.

Los derechos de PI en la empresa estatal socialista cubana refieren a títulos de propiedad sobre bienes intangibles patrimoniales de estas entidades que se obtienen en vínculo laboral con autonomía para ejercer sus atribuciones en la gestión económica y relaciones de intercambio frente a terceros en el comercio y utiliza como medio de lograr metas concretas de eficiencia, productividad, competitividad para alcanzar los fines para los cuales fueron creadas por el Estado en representación de los intereses supremos del pueblo: contribuir con sostenibilidad al incremento de las aportaciones a la sociedad y al cuidado del medio ambiente.

El despliegue consciente y ordenado de estrategias de PI, derecho de autor y buenas prácticas de uso en la empresa estatal socialista cubana contribuiría al ciclo completo de innovación en armonía con la ANIR y el Fórum de Ciencia y Técnica como organizaciones de masas y políticas autóctonas que promueven la protección y difusión participativa interna, fortaleza en la identificación de la competitividad y alto desempeño de trabajadores individuales y colectivos laborales innovadores comprometidos con las transformaciones de la sociedad.

El establecimiento de actividades de I+D y la gestión de ciencia y tecnología por proyectos en el sector empresarial estatal cubano posibilitarán la planificación socialista de producción y ordenamiento contable para la valorización y registro de activos intangibles de PI.

La valoración y registro contable de activos de PI posibilitará que estos se incluyan en los estados financieros organizacionales y que se evidencie la posición financiera a partir de considerar también el patrimonio intangible empresarial.

El uso de nuevas formas jurídicas organizativas del sector empresarial, universidades y centros de investigación que posibiliten incentivar la investigación 
que demanda el país requiere de respaldo jurídico para la realización de alianzas estratégicas entre los diferentes sectores que promuevan la innovación y registro de derechos de PI con enfoque inclusivo o social.

El estudio de empresas de base tecnológica y su regulación en Cuba acorde con las necesidades del país resultan necesario en sectores económicos priorizados y de punta como la industria biotecnológica y farmacéutica.

La definición de políticas públicas que promuevan el ciclo completo de innovación en Cuba, con inclusión de la protección y difusión estratégica de los resultados en relaciones entre partes, constituyen demandas actuales para el debido equilibrio con las necesidades e intereses del país y la armonización de buenas prácticas y usos de la PI en la comercialización a nivel local, nacional y en nichos de mercado internacionales. En ello resulta imprescindible una nueva normativa en materia de ciencia, tecnología e innovación.

\section{BibliografíA}

Buitrago Díaz, Esperanza, "Propiedad intelectual y desarrollo tras el acuerdo sobre los ADPIC", Propiedad Intelectual, Mérida-Venezuela, n. ${ }^{\circ} 12$, año VIII, 2009, disponible en: http://www.saber.ula.ve/bitstream/123456789/30458/1/ articulo3.pdf [16/12/2014].

Busaniche, Beatriz, "A 20 años de la firma de los adpic. Un debate pendiente sobre la propiedad intelectual y el desarrollo en América Latina”, Fundación Vía Libre, 2015, disponible en: http://www.vialibre.org.ar/wp-content/ uploads/2015/04/20anios.adpic_..pdf [26/12/2015].

Castro Ruz, Fidel, "Discurso pronunciado por Fidel Castro Ruz, Presidente de la República de Cuba, en la clausura del XI Foro de Ciencia y Técnica efectuada en el Palacio de las Convenciones el 21 de diciembre de 1996", XI Foro de Ciencia y Técnica, 1996, disponible en: http://www.cuba.cu/gobierno/ [20/05/2016].

Castro Ruz, Fidel, "Mentiras deliberadas, muertes extrañas y agresión a la economía mundial. Reflexiones del Comandante en Jefe (18 de septiembre 2007)", Granma, año 18, n.o 70, 11 de marzo de 2014, disponible en: http:// www.granma.cu/granmad/secciones/reflexiones/esp-049.html [05/01/2017]-

Comité Ejecutivo del Concejo de Ministros, Decreto n. ${ }^{\circ} 120$, Reglamento de la Ley de Innovaciones y Racionalizaciones, Consejo de Ministros, La Habana, 1984.

Consejo de Estado, Decreto-Ley n. ${ }^{\circ} 203$ de marcas y otros signos distintivos, Gaceta Oficial de la República de Cuba, 1999.

Consejo de Estado, Decreto-Ley n. ${ }^{\circ} 228$, de las indicaciones geográficas, n. ${ }^{\circ} 11$, Gaceta Oficial de la República de Cuba, 2002.

Consejo de Estado, Decreto Ley n. ${ }^{\circ} 290$, de las invenciones, dibujos y modelos industriales, n. ${ }^{\circ}$ 2, Gaceta Oficial de la República de Cuba, 2012, disponible en: http://www.gacetaoficial.cu 
Consejo de Estado, Decreto Ley n. 291 , de las variedades vegetales, n. o 2, Gaceta Oficial de la República de Cuba, 2012, disponible en: http://www.gacetaoficial.cu Consejo de Estado, Decreto Ley n. ${ }^{\circ} 292$, de esquema de trazado de circuito integrado, n. ${ }^{\circ}$ 2, Gaceta Oficial de la República de Cuba, 2012, disponible en: http://www.gacetaoficial.cu, 2012.

CEPAL, Ciencia, tecnología e innovación en la economía digital. La situación de América Latina y el Caribe, n. ${ }^{\circ}$ S.16-00833, Equipo de la Unidad de Innovación y Nuevas Tecnologías de la División de Desarrollo Productivo y Empresarial de la Comisión Económica para América Latina y el Caribe, Segunda Reunión de la Conferencia de Ciencia, Innovación y Tecnologías de la Información y las Comunicaciones de la CePAL que se celebrará en San José del 12 al 13 de septiembre, 2016.

Ciencia, Tecnología y Medio Ambiente (Ministerio), Resolución n. ${ }^{\text {2 21, Sistema }}$ Nacional de Propiedad Industrial de la República de Cuba, 2003, disponible en: http://www.ocpi.cu/sites/default/files/archivos_a_vincular/resol21.pdf [7/10/2009].

Consejo de Ministros, Ley n. 38 De las innovaciones y racionalizaciones, $n .{ }^{\circ} 38$, (Impresa ed.), Comité Ejecutivo, La Habana, Cuba, 1984.

Consejo de Ministros, Decreto n. ${ }^{\circ}$ 281/2007, Reglamento para la implantación y consolidación del sistema de dirección y gestión empresarial estatal, versión actualizada, revisada y concordada, 2014, disponible en: http://www.gacetaoficial.cu/ [15/01/2015]-

Contreras Villavicencio, Dulce María, "Propiedad industrial para ingeniería hidráulica. Asignatura optativa en la formación de profesionales en Villa Clara", REGIE, Revista Jurídica de Investigación e Innovación Educativa, junio, 2015, disponible en: http://www.eumed.net/rev/rejie [3/7/2015].

Contreras Villavicencio, Dulce María y Suárez Gutiérrez, Evelio, Estrategias de mercadotecnia integradas con propiedad intelectual para potenciar el patrimonio intangible empresarial, trabajo presentado en el 9. ${ }^{\text {a Conferencia }}$ Internacional de Ciencias Empresariales, Complejo de Turismo Topes de Collantes, Trinidad, Editorial Samuel Feijóo, Santi Spiritus, 2014.

Contreras Villavicencio, Dulce María y Suárez Gutiérrez, Evelio, Propiedad industrial, ciencia, tecnología e innovación en alianzas universidad-empresa, trabajo presentado en el VI Encuentro de Tecnologías y Gestión del Conocimiento TeCNOGesc 2016 que se celebró los días 20 y 21 de octubre, Ciudad de Santa Clara, Editorial Samuel Feijóo, uclv citma. Memorias del Evento en CD, Villa Clara, 2016.

Contreras Villavicencio, Dulce María; Suárez Gutiérrez, Evelio y Recarey Morfa, Carlos A., "Estrategias de propiedad industrial en convenios de integración y cooperación universidad-empresa en Villa Clara", Revista Caribeña de Ciencias Sociales, marzo, 2016, disponible en: http://www.eumed.net/rev/ caribe/2016/03/cooperacion.html [16/03/2016]. 
De León, Ignacio L. y Fernández Donoso, José, "El uso de la Propiedad Intelectual en el desarrollo de los mercados de innovación", 2016, disponible en: http://s3.amazonaws.com/academia.edu.documents/37520389/Mercados_Innovacion_LATAM_V0405.pdf?AWSAccessKeyId=AKIAIWOWYYGZ2Y53UL3A\&Ex pires $=1491924089 \&$ Signature $=$ auNlfvnGcPLhMwmgFSLLgLrnUzA\%3D\&r esponse-content-disposition=inline\%3B\%20filename\%3DEl_uso_de_la_Propiedad_Intelectual_en_el.pdf [28/12/2016].

De las Heras, Elena et al., "El tratamiento contable de los gastos de I+D", Actualidad Contable, Recopilación de casos prácticos tratados, AECA Asociación Española de Contabilidad y Administración de Empresas, 2015, disponible en: http://www.elcriterio.com/casos_practicos_newsletter.pdf [22/01/2015].

Fabelo Corzo, José Ramón, "Conocimiento y valor. Parte iII: La dialéctica materialista: concepción y método”, en Suárez, L. et al., Filosofía marxista, t. I, La Habana, Editorial Félix Varela, Ministerio de Enseñanza Superior, Dirección de Marxismo-Leninismo, 2009.

Fernández Bulté, Julio, "Prólogo", en Lillian Álvarez Navarrete, Derecho de ¿autor? El debate de hoy, Royma Caña ed., Editorial de Ciencias Sociales, La Habana, 2008.

Gouvea, Raul, Kassicieh, Sul y Montoya, M. J. R., "Using the quadruple helix to design strategies for the green economy", Technological Forecasting \& Social Change, 2012, disponible en: http://dx.doi.org/10.1016/j.techfore.2012.05.003 [6/01/2014].

Guevara de la Serna, Ernesto "Che", "Discurso en la clausura del Encuentro Internacional de estudiantes de arquitectura", en Guevara, Ernesto, Colección Nuestra América. Ernesto Che Guevara. Obras 1957-1967, t. II, La transformación politica, económica y social, Casa de las Américas, La Habana, 1970.

Hernández Sampieri, Roberto; Fernández Collado, Carlos y Baptista Lucio, Pilar, Metodología de la Investigación, $4 .^{\mathrm{a}}$ ed., México, McGraw-Hill Interamericana, 2006.

Horta Herrera, Emilia et al., "Cambios en el ordenamiento jurídico cubano a la luz del Acuerdo sobre los Derechos de Propiedad Intelectual relacionados con el Comercio, incluido el comercio de mercancías falsificadas, TRIPs, de la Organización Mundial del Comercio y su influencia en la estrategia de protección y comercialización de los resultados de los centros del Polo Científico", en Moreno Cruz, Marta Milagros y Horta Herrera, Emilia, Selección de lecturas de propiedad industrial, t. II, Editorial Félix Varela, La Habana, 2003.

Kalanje, Christopher, "Aprovechar al máximo el sistema de propiedad intelectual: algo más que el derecho a excluir", disponible en: http://www.wipo.int/ sme/es/documents/leveraging_ip.htm [15/01/2016].

Kim, Younghwan; Kim, Wonjoon y YAng, TAeyong, "The effect of the triple helix system and habitat on regional entrepreneurship: empirical evidence from the U.S”, 2012, disponible en: http://www.elsevier.com/locate/respol [6/01/2014]. 
Lage DÁvila, Agustín, "Las biotecnologías y la nueva economía: crear y valorizar los bienes intangibles", Revista de Biotecnología Aplicada, n. 3 3, vol. 17, 2000, disponible en: http://www.bioline.org.br/request?ba00018 [26/02/2008].

Lage Dávila, Agustín, "La economía del conocimiento y el socialismo: ¿Hay una oportunidad para el desarrollo?", Cuba Socialista. Revista Teórica y Política, n. ${ }^{\circ} 41,2006$, disponible en: http://www.cubasocialista.cu/texto/enlaces.html [26/02/2008].

Lage Dávila, Agustín, "Cuba ha creado las bases para el tránsito a una economía basada en el conocimiento", Cuba Socialista. Revista Teórica y Política, octubre, 2007, disponible en: http://www.cubasocialista.cu [22/06/2009].

Lage Dávila, Agustín, "Las funciones de la ciencia en el modelo económico cubano", Temas, n. ${ }^{\circ}$ 69, enero-marzo, 2012, disponible en: http://www.rebelion. org/mostrar.php?tipo=5\&id=Agust\%EDn\%20Lage\&inicio=0 [15/10/15] .

Lage DÁvila, Agustín, La economía del conocimiento y el socialismo, Aldo Gutiérrez Rivera ed., Sello Editorial Academia, La Habana, 2013.

Lage Dávila, Agustín, “¿Una empresa estatal socialista de alta tecnología?-Cuba”, en el weblog Jaime Lago, disponible en: http://www.jaimelago.org/node/83 [26/12/2015].

Mantilla Cárdenas, William, "Las perspectivas de la investigación social del derecho en el campo de la propiedad intelectual y las nuevas tecnologías", La Propiedad Inmaterial, n. ${ }^{\circ}$ 3, 2001, disponible en: http://www.uexternado.edu. co/propiedadinmaterial [17/04/15].

Mantilla Cárdenas, William, "Fragmentos de filosofía de la ciencia y su relación con trayectos de investigación sociojurídica para la producción de conocimiento en el dominio disciplinar de la propiedad intelectual", La Propiedad Inmaterial, n. ${ }^{\circ}$ 6, enero-junio, 2003, disponible en: http://www.uexternado.edu.co/ propiedadinmaterial [17/04/15].

Martínez Martínez, Osvaldo, Crisis global y pensamiento del Ché sobre economía internacional, La Habana, Ciencias Sociales, Ruth Casa Editorial, 2009.

Matheus Osechas, Alida Sabrina, "Propiedad, ejercicio y titularidad de los derechos intelectuales en la normativa laboral venezolana”, Propiedad Intelectual, Mérida-Venezuela, año XIII, n. ${ }^{\circ}$ 17, enero-diciembre, 2014, disponible en: http://www.saber.ula.ve/bitstream/123456789/39204/1/articulo1.pdf [8/10/2015].

Ministerio de Finanzas y Precios, Resolución n. ${ }^{\circ}$ 083, Establece requisitos para las empresas estatales, sociedades mercantiles, civiles de servicios, interesadas en ejercer la actividad de valuación de activos, n. ${ }^{\circ}$ 16/12, Gaceta Oficial de la República de Cuba, 2012.

Mora, Araceli, "Activos Intangibles generados internamente por la empresa: Los Gastos de I+D", Actualidad Contable, Recopilación de casos prácticos tratados, AECA Asociación Española de Contabilidad y Administración de Empresas, 
2015, disponible en: http://www.elcriterio.com/casos_practicos_newsletter. pdf [22/01/2015].

Morejón Borjas, Martha María, “Tecnología para la gestión de la propiedad intelectual en la empresa estatal cubana. Aplicación en organizaciones empresariales de la Provincia Holguín", tesis presentada en opción al título de Doctor en Ciencias Técnicas, Instituto Superior de Tecnologías y Ciencias Aplicadas, Cuba, Ciudad de Holguín, 2012.

Moreno Linde, Manolo, "Metodología de la elaboración de trabajos de investigación jurídica desde un enfoque práctico. El valor de la experiencia profesional", REGIE. Revista Jurídica de Investigación e Innovación Educativa, n. ${ }^{\circ} 11$, enero, 2015, disponible en: http://www.eumed.net/rev/rejie/11/index.htm [3/7/2015].

Núñez Jover, Jorge, "Prólogo", en Castro Díaz-Balart, Fidel, Ciencia, tecnología y sociedad. Hacia un desarrollo sostenible en la Era de la Globalización, 2. ${ }^{\mathrm{a}}$ ed., Instituto Cubano del Libro, Editorial Científico-Técnica, La Habana, 2004.

Núñez Jover, Jorge, La ciencia y la tecnología como procesos sociales. Lo que la educación científica no debería olvidar, 2. ${ }^{\mathrm{a}}$ ed., La Habana, Félix Varela, 2007.

NúNEz Jover, Jorge, Innovación y desarrollo social: un reto para CTS, La Habana, 2010.

Núñez Jover, Jorge y Figueroa Alfonso, Galia, "Biotecnología y sociedad en Cuba: el caso del Centro de Inmunología Molecular", Trilogía. Ciencia, Tecnología y Sociedad, n. ${ }^{o}$ 10, enero-junio, 2014, disponible en: http://itmojs. itm.edu.co/index.php/trilogia/article/viewFile/587/pdf_7 [15/01/2015].

Núñez Jover, Jorge; Montalvo, Luis Félix y Pérez, Isarelis, Nueva universidad, conocimiento y desarrollo social basado en el conocimiento, Universidad de La Habana, La Habana, 2006.

OMPI, Informe sobre la propiedad intelectual en el mundo. Los nuevos parámetros de la innovación, 2011, disponible en: http://www.wipo.int/ [11/11/2014].

OMPI, Informe mundial sobre la propiedad intelectual. Reputación e imagen en el mercado global, 2013, disponible en: http://www.wipo.int/ [28/01/2015].

OMPI, Reseñas de los convenios, tratados y acuerdos administrados por la OMPI, publicación OMPI n. ${ }^{\circ} 442 S, 13 .^{a}$ ed., Ginebra, 2013, disponible en: www.wipo.int

Orozco Silva, Eduardo, "La inteligencia empresarial como herramienta de la innovación", conferencia virtual, "Universidad para todos", ciclo de conferencias televisadas en el curso Nuevas Tecnologías, Clase n. ${ }^{\circ}$ 12, Instituto de Información Científica y Tecnológica (IDICT), La Habana, 2007.

Partido Comunista de Cuba, Lineamientos de la Política Económica y Social del Partido y la Revolución, n. ${ }^{\circ}$ especial, Editora Política del CCPCC, La Habana, 2011.

Partido Comunista de Cuba, Actualización de los Lineamientos de la Política Económica y Social del Partido y la Revolución para el periodo 2016-2021 aprobados en el 7 mo. Congreso del Partido en abril de 2016 y por la Asamblea Nacional 
del Poder Popular en julio de 2016, n. ${ }^{\circ}$ especial, Editora Política del CCPCC, La Habana, 2016.

Partido Comunista de Cuba, Informe Central al 7 mo. Congreso del Partido Comunista de Cuba, presentado por el Primer Secretario del Comité Central, General de Ejército Raúl Castro Ruz, La Habana, 16 de Abril, 2016, disponible en: http:// www.pcc.cu/ [20/05/2016].

Partido Comunista de Cuba, Plan Nacional de Desarrollo Económico y Social hasta 2030: propuesta de visión de la nación, ejes y sectores estratégicos, 2016, disponible en: http://www.pcc.cu/pdf/congresos_asambleas/vii_congreso/conceptualizacion.pdf [22/12/2016].

Pérez Almaguer, Andrés Francisco, "Competitividad. Una mirada en el proceso de comercialización empresarial”, Revista Caribeña de Ciencias Sociales, junio, 2015, disponible en: http://www.eumed.net/rev/caribe/2015/06/competitividad.html [28/04/2016].

Pérez Hernández, Lissette, "Introito”, en Carlos Manuel Villabella Armengol, Investigación y comunicación científica en la ciencia jurídica, La Habana, Editorial Universitaria Félix Varela y Ediciones Universidad de Camagüey, 2012.

PItKethly, Robert, "Capítulo 2.2. Una estrategia de propiedad intelectual (PI)", en P. Anguita et al., Gestión de la propiedad intelectual innovación en agricultura $y$ en salud: un manual de buenas prácticas, FIA: Programa FIA-PIPRA (Chile) y PIPRA (USA), 2010, disponible en: http://www.fia.pipra.org

Ramírez Cabrera, Zahyly y Moreno Cruz, Martha Milagros, "El trips. Su influencia en la legislación cubana", Revista Cubana de Derecho, n. ${ }^{\circ} 12$, enero 1997- diciembre 1998, pp. 4-28.

Rengifo García, Ernesto, "Estudio sobre patentes y dominio público", La Propiedad Inmaterial, n. ${ }^{\circ}$ 15, 2011, disponible en: http://www.uexternado.edu. co/propiedadinmaterial http://revistas.uexternado.edu.co/index.php/propin/ issue/view/316 [10/01/2012].

Romeu Lameiras, Eva, "La vigilancia tecnológica y la propiedad industrial en la gestión del nuevo modelo económico cubano", trabajo presentado en el VI Encuentro de Tecnología y Gestión del Conocimiento TeCNOGesc 2014 que sesionó del 22 al 23 de octubre, Convenciones Bolívar, Centro de Información y Gestión Tecnológica de Villa Clara, Santa Clara, Villa Clara, Cuba, 2014.

Strand, Oivind y LeYdesdorfF, Loet, "Where is synergy indicated in the Norwegian innovation system? Triple-Helix relations among technology, organization, and geography", Technological Forecasting \& Social Change, 2012, disponible en: http://dx.doi.org/10.1016/j.techfore [6/01/2014].

Suárez Arroyo, Benjamín, La innovación tecnológica y los paradigmas sociales, Barcelona, Icaria, 2008, disponible en: http://www.icariaeditorial.com

Suárez Gutiérrez, Evelio y Contreras Villavicencio, Dulce María, Contabilidad de activos intangibles de propiedad intelectual. Necesidad en la empresa estatal socialista cubana, Copyright OmniScriptum GmbH \& Co. 
KG ed., Editorial Académica Española, 2016, disponible en: http://www. eae-publishing.com

Títu, Minail Aurel et al., "The Place and the Role of the Intellectual Property Assets in the Knowledge Based Organization Context", Balkan Region Conference on Engineering and Business Education, ISSN (Online) 2391-8160, DOI: 10.1515/cplbu-2015-0027, vol. 1, n. ${ }^{\circ}$ 1, January, 2016, disponible en: http://www.degruyter.com/view/j/cplbu.2015.1.issue-1/cplbu-2015-0027/ cplbu-2015-0027.xml [27/12/2016].

Triana Cordovi, Juan, "Cuba: desarrollo social, globalización y economía del conocimiento", conferencia de clausura en el IV Taller Internacional de Inteligencia Empresarial y Gestión del Conocimiento en la Empresa (IntEmpres 2003) que sesionó en el Hotel Nacional del 29 de noviembre al 3 de diciembre de 2003, Instituto de Información Científica y Tecnológica (IDICT) del Ministerio de Ciencia, Tecnología y Medio Ambiente de la República de Cuba, La Habana, 2003.

Triana Cordovi, Juan, "Cuba: ¿¿de la "actualización" del modelo económico al desarrollo?", Nueva Sociedad, n. ${ }^{\circ}$ 242, noviembre-diciembre, 2012, pp. 82-91.

Unión Europea, Estrategia sobre los derechos de propiedad industrial, 2008, disponible en: http://www.ask.com/web?q=Comunicaci\%c3\%b3n+de+la+Comisi\%c3\% b3n+al+Parlamento+Europeo\%2c\&o=\&l=\&qsrc $=[10 / 03 / 2014]$.

Vázquez de Alvaré, Dánice de la Caridad y Moreno Cruz, Marta Milagros, "Breves comentarios sobre la nueva regulación de invenciones en Cuba", Revista Cubana de Derecho, n.o 41, enero-junio, 2013, pp.19-42.

Vicente Oliva, Silvia; Martínez Sánchez, Ángel y Berges Muro, Luis, "Buenas prácticas en la gestión de proyectos de $\mathrm{I}+\mathrm{D}+\mathrm{i}$, capacidad de absorción de conocimiento y éxito", Dyna, vol. 82, n. ${ }^{\circ} 191,2015$, disponible en: http:// www.redalyc.org/articulo.oa?id=49639089014 [26/12/2015].

Villabella Armengol, Carlos Manuel, Investigación y comunicación cientifica en la ciencia jurídica, Ernesto Piñero de Laosa ed., La Habana, Editorial Universitaria Félix Varela y Ediciones Universidad de Camagüey, 2012.

Xing, Yan; Ge, Zhangzhi y Song, Wei, "Research on Innovation of Science and Technology Investment and Financing of smes in Intellectual Property", Revista Technology and Investment, n. ${ }^{\circ}$ 7, mayo, 2016, disponible en: http://www.file. scirp.org/pdf/TI_2016050914380856.pdf [3/06/2016].

Yallwe, Alem Hagos y Buscemi, Antonino, "An era of intangible assets", Journal of Applied Finance \& Banking, vol. 4, n. ${ }^{\circ}$ 5, 2014, disponible en: http://www. scienpress.com/Upload/JAFB/Vol\%204_5_2.pdf [15/01/2015]. 